\title{
HRTEM Study of Co Nanoparticles Fabricated by a Cluster Gun Technique
}

\author{
Y. Zhang, P. Liu, and G. C. Hadjipanayis \\ Department of Physics and Astronomy, University of Delaware, Newark, DE 19716
}

In recent years, ferromagnetic metal-insulator granular films have attracted much attention, because of their potential applications in tunnel magnetoresistance (MR) and high density information storage media [1-2]. Cluster guns have been found to be suitable for the fabrication of nanoparticles in a wide range of materials because they allow the formation of magnetic nanoparticles directly inside the sputtering chamber, thus reducing oxidation and sintering, which are often observed in annealed samples prepared by the multilayer precursor technique [3-4].

In this study, Co nanoparticles embedded in a carbon matrix were fabricated by a cluster gun technique with different sputtering power and times, and were deposited on a $\mathrm{Cu}$ TEM grid. A JEOL JEM 3010 microscope operating at $300 \mathrm{kV}$ was used to characterize the TEM/HRTEM microstructure of Co nanoparticles.

Figure 1 shows TEM microstructure of Co nanoparticles fabricated with different sputtering power and times. Electron diffraction patterns indicated an fcc $\mathrm{Co}(\mathrm{a}=0.3545 \mathrm{~nm}, \mathrm{Fm} \overline{3} \mathrm{~m})$ phase structure in these samples. At a low sputtering power and a sputtering time of 3s (Fig.1a), the sample shows isolated Co nanoparticles with a low density, having a narrow particle size distribution centered at 6 $\mathrm{nm}$. With increasing sputtering power, both the particle size and density of Co nanoparticles increase, and most of the Co nanoparticles are in form of clusters (Fig. 1b). With increasing sputtering time at a fixed sputtering power, the density of nanoparticles gradually increases with increasing sputtering time at a fixed sputtering power, while the particle size slightly increases (Figs. $1 \mathrm{c} \& 1 \mathrm{~d})$. Co nanoparticles tend to form clusters after longer sputtering time, although some isolated nanoparticles can still be observed. The isolated Co nanoparticles in this study show different morphology. Figure $2 \mathrm{a}$ shows a Co nanoparticle with a triangle shape. Figure $2 \mathrm{~b}$ shows a Co nanoparticle with a hexagonal shape. Figure $2 \mathrm{c}$ shows two spherical Co nanoparticles, which are adjacent and have their own crystallographic orientations. However, irregular Co nanoparticles are also observed (Fig. 2d). Actually, all these Co nanoparticles with different morphology are in form of single crystalline structure. The clusters of Co nanoparticles are also studied. HRTEM shows that these clusters are either elongated nanoparticles or agglomerated nanoparticles (Fig. 3). Elongated nanoparticles are single crystalline, whereas agglomerated nanoparticles are polycrystalline. Therefore, cubic Co nanoparticles with different morphology have been successfully fabricated by using the cluster gun technique [5].

\section{References}

[1] J. L. Gittleman, Y. Goldstein, and S. Bozowski, Phys. Rev. B 5, (1972) 3609.

[2] T. Furubayashi and I. Nakatani, J. Appl. Phys. 79, (1996) 6258.

[3] V. Skumryev, S. Stoyanov, Y. Zhang, et al., Nature (London) 423, (2003) 850.

[4] S. Stoyanov, Y. Huang, Y. Zhang, et al., J. Appl. Phys. 93, (2003) 7190.

[5] This work is supported by NSF DMR Grant No. 0302544. 

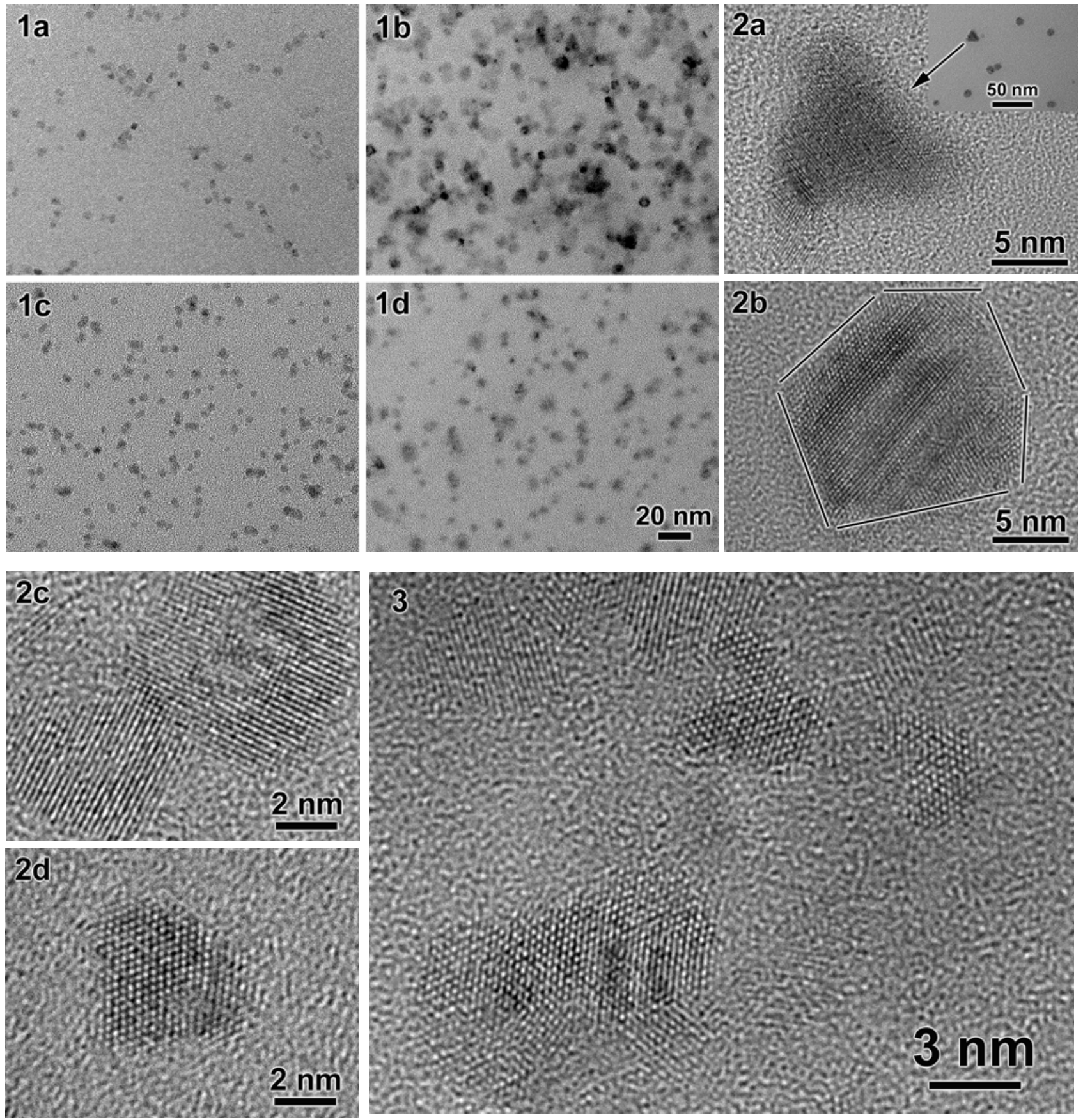

Fig.1. TEM microstructure of Co nanoparticles fabricated with different sputtering parameters: (a) Sputtered for $3 \mathrm{~s}$ at a low sputtering power (37W); (b) $3 \mathrm{~s}$ at a high sputtering power (72W); (c) $5 \mathrm{~s}$ at a low sputtering power $(29 \mathrm{~W})$; (d) 15 s at a low sputtering power $(29 \mathrm{~W})$.

Fig.2. Co nanoparticles with different cross-sectional morphology: (a) Triangle; (b) Hexagonal; (c) Spherical; (d) Irregular.

Fig.3. HRTEM showing a cluster of Co nanoparticles and isolated Co single nanoparticles. 